\title{
Modulation of the TGF- $\beta$ signaling pathway by long noncoding RNA in hepatocellular carcinoma
}

\author{
Mengzhen $\operatorname{Han}^{1,2+}$, Zhibin Liao ${ }^{1,2+}$, Furong Liu ${ }^{1,2}$, Xiaoping Chen ${ }^{1,2^{*}}$ and Bixiang Zhang ${ }^{1,2^{*}}$
}

\begin{abstract}
Hepatocellular carcinoma (HCC) is a type of liver cancer with poor prognosis. There have been demonstrated to exist many possible mechanisms in HCC tumorigenesis, and recent investigations have provided some promising therapy targets. However, further mechanisms remain to be researched to improve the therapeutic strategy and diagnosis of HCC. Transforming growth factor- $\beta$ (TGF- $\beta$ ) is a pleiotropic cytokine which plays critical roles in networks of different cellular processes, and TGF- $\beta$ signaling has been found to participate in tumor initiation and development of HCC in recent years. Moreover, among the molecules and signaling pathways, researchers paid more attention to IncRNAs (long non-coding RNAs), but the connection between IncRNAs and TGF-Bremain poorly understood. In this review, we conclude the malignant procedure which IncRNAs and TGF- $\beta$ involved in, and summarize the mechanisms of IncRNAs and TGF-Bin HCC initiation and development. Furthermore, the interaction between IncRNA and TGF- $\beta$ are paid more attention, and the potential therapy targets are mentioned.
\end{abstract}

Keywords: Long noncoding RNA, TGF- $\beta$, Hepatocellular carcinoma, Therapy target

\section{Introduction}

Hepatocellular carcinoma (HCC), which accounts for 70$90 \%$ of primary liver cancer, is still the leading cause of cancer-related death worldwide, particularly in China [1, 2]. Most of HCC patients have suffered from cirrhosis caused by chronic hepatitis $B$ and hepatitis $C$ virus infection [3]. However, the molecular mechanisms in HCC pathogenesis remain not completely understood [4]. Early diagnosis is difficult for $\mathrm{HCC}$, because there is always no obviously symptom in early stages of HCC. Although abundant therapeutic methods have been adopted, including surgical resection, chemotherapy, and targeted therapy, the prognosis of HCC is still poor [5]. In recent years, researches aimed at the roles of lncRNAs and TGF- $\beta$ in

\footnotetext{
* Correspondence: chenxpchenxp@163.com; bixiangzhang@163.com ${ }^{\dagger}$ Mengzhen Han and Zhibin Liao contributed equally to this work. 'Hepatic Surgery Center, Tongji Hospital, Tongji Medical College, Huazhong University of Science and Technology, 1095 Jiefang Avenue, Wuhan 430030, China

Full list of author information is available at the end of the article
}

HCC tumorigenesis. The results have also provided some possible therapy targets of HCC.

Transforming growth factor (TGF)- $\beta$ is a multifunctional cytokine which has important effects on regulating cell growth, apoptosis and differentiation [6]. It belongs to the TGF- $\beta$ superfamily, which consist of several related molecules, such as TGF $\beta$ s (TGF $\beta 1$, TGF $\beta 2$ and TGF $\beta 3$ ), bone morphogenic proteins (BMPs), activins, growth and differentiation factors (GDFs) and anti-mullerian hormone $(\mathrm{AMH})[7,8]$. Members of TGF- $\beta$ superfamily pass signal through membrane-associated serine/threonine kinase receptors, and then activate SMAD transcription factors as well as other downstream molecules [9]. TGF- $\beta$ proteins and their downstream molecules constitute the TGF- $\beta$ signaling pathway, which attract many researchers to investigate its function.

Previous studies has indicated noncoding RNAs, containing microRNA (miRNA), circular RNA (circRNA), PIWI-interacting RNA (piRNA) and particularly long 
noncoding RNA (lncRNA), could participate in distinct physiological and pathological processes [10]. IncRNAs have been discovered to promote or inhibit some gene expression through diverse mechanisms [11]. In this review, we mainly concentrate on TGF- $\beta$ signaling pathway, lncRNAs and their connection in HCC, which may provide some novel strategies for therapy and early diagnosis.

\section{TGF- $\beta$ signaling pathway and its function in HCC}

TGF- $\beta$ signaling pathway can regulate a variety of cellular processes in human. TGF $\beta$ s are important molecules in this signaling pathway. Before theirmaturation, TGF $\beta s$ undergo several posttranslational modifications from precursor proteins of 390-412 amino acids. Firstly, two precursor proteins assemble to a dimer and subsequent cleaved by furin endopeptidase. After this process, latency-associated peptide (LAP) and the mature TGF- $\beta$ protein come into being. Then, the LAP surround mature TGF- $\beta$ and these two molecules form the small latent complex (SLC). Finally, SLC can bind to latent TGF- $\beta$ binding protein (LTBP) via disulfide bonds, which is important in TGF- $\beta$ proteins stability, assembly and secretion $[12,13]$.

After released into extracellular matrix, the TGF- $\beta$ dimers bind to type II receptor and then type I receptor combines with type II receptor, which leads to the phosphorylation of type I receptors GS domain, SMAD2 and 3 . Additionally, other branch of TGF- $\beta$ superfamily like BMPs can bind to receptors and phosphorylate SMAD 1, 5 and 8 which are known as R-SMADs [14]. Furthermore, phosphorylated R-SMAD and SMAD4 form heteromeric complexes and then translocated into the nucleus [15]. Meanwhile, there are inhibitory SMAD proteins include SMAD6 and SMAD7 (I-SMADs), which are activated by TGF- $\beta$ as a negative feedback system [16]. Taken together, the SMADs mentioned above make up the canonical TGF- $\beta$ signaling pathway. However, there are some noncanonical downstream mediators which are induced by TGF- $\beta$ independent on SMADs proteins, for example extracellular-signal regulated kinase (ERK), p38 mitogen-activated protein kinase (p38 MAPK), PI3k/AKT, RING domain-containing E3 ubiquitin ligases TRAF6, and several small GTPase such as Rho1, Rac and KRAS [17-21].

Disruption of TGF- $\beta$ signaling pathway contributes to many diseases including all kinds of cancers [22]. In prior studies, abundance of results showed the TGF- $\beta$ signaling pathway played pleiotropic roles in HCC, and exerts functions of either tumor suppressor or promoter [23]. In early stages HCC cells, activation of TGF- $\beta$ lead to anti-proliferation response by inhibiting cell cycle at G1/S, or regulating p21 and p15 [24]. On the other hand, the overexpression of TGF $\beta$ s in HCC suggests the malignant potential in complicated processes [25].

Firstly, TGF- $\beta$ signaling participates in the process of cirrhosis and liver cancer initiation. Hepatic progenitor cells (HPCs) are stem cells in liver which can be activated under the circumstances of chronic liver injury. Recent studies indicated that HPCs could autocrine TGF $\beta 1$ and induce epithelial mesenchymal transition states through activation of SMAD2 and 3 in canonical signaling pathway. Furthermore, TGF $\beta 1$ secreted by HPCs can also activate autonomous secretion of connective tissue growth factor (CTGF) mediated by SMAD independent signaling, such as ERK, JNK and p38 MAPK signaling. Both of the mechanisms in HPCs activation are related to liver fibrosis and hepatocarcinogenesis [26, 27]. Likewise, known as stem cells of HCC, liver cancer stem cells (LCSCs) are also called tumorinitiating cells, which display abilities of self-renewal and differentiation and participate in new tumors generation [28]. In recent researches, it was reported that tumorassociated macrophages (TAMs) could secret TGF $\beta 1$ to promote LCSC properties by inducing EMT [29], and tumor-associated neutrophils (TANs) could secret TGF $\beta 2$ and BMP2, which induce miR-301b-3p and enhance stem cell characteristics in HCC [30]. Liver fibrosis is the most common precancerous change of HCC, and hepatic stellate cells (HSCs) have been identified as important myofibroblast progenitor cells in liver fibrosis. The TGF- $\beta$ signaling pathway is involved in emergency of HSCs and the production of several extracellular matrix proteins. It was reported that bromodomaincontaining protein 4 (BRD4) was critical in activation of HSCs, while experiments in murine models showed inhibitor of BRD4 could attenuate liver fibrosis and tumorigenesis through repressing TGF- $\beta$ signaling pathway $[31,32]$. These results indicated a tight connection between TGF- $\beta$ and HCC initiation.

Secondly, TGF- $\beta$ signaling also plays crucial roles in epithelial-mesenchymal transition (EMT). EMT is a process in which epithelial cells lose their adhesions between each other and require more mesenchymal characteristic. Meanwhile, it could be observed that some epithelial-typed proteins (E-cadherin, cytokeratin) are attenuated and some mesenchymal markers such as vimentin and $\mathrm{N}$-cadherin are upregulated [33]. Therefore, EMT is closely associated with tumor metastasis [34, 35]. In previous studies, multiple findings have revealed that the process of EMT is regulated by the EMT-transcription factors (EMT-TFs) such as SNAIL, TWIST and ZEB1/2, which were reported as important inducers of $\mathrm{HCC}[36,37]$. The receptor tyrosine kinase Axl is upregulated in HCC cells. Axl can cause phosphorylation of SMAD3 linker region by binding to 14$3-3 \zeta$, and result in upregulation of many downstream 
molecules of TGF- $\beta$ signaling like Snail and MMP9 [38]. Platelet-derived growth factor (PDGF) and $\beta$-catenin are important hallmarks of EMT. Recent studies reported that TGF- $\beta$ could induce autocrine and secretion of PDGF, then activate PI3K and $\mathrm{Wnt} / \beta$-catenin signaling upon hepatocellular EMT [39]. Moreover, Protein Tyrosine Phosphatase Receptor Epsilon (PTPRe) which can bind to SMAD3 upon TGF- $\beta$ stimulation, was observed to recruit SMAD3 to TGFBRI and induce EMT in HCC [40]. Overall, TGF- $\beta$ signaling is the key inducer of EMT in HCC by abundant of pathways, including many different mechanisms of tumor progression.

Thirdly, since dysregulation of the immune system has been frequently reported in HCC, TGF $\beta$ s are indicated as anti-inflammatory cytokines to inhibit anti-tumor immune responses [41]. TGF- $\beta$ is critical for regulating immune cells differentiation, proliferation and development, including myeloid-derived suppressor cells (MDSCs), tumor-associated macrophages (TAMs), NK cells, and dendritic cells (DCs) [42]. Treg cells mainly play the inhibitory roles in $\mathrm{HCC}$ and lead to suppression of anti-tumor responses [43]. TGF- $\beta$ can regulate Treg cells activity, and inhibit immune responses through suppressing effector T cells such as CD8+ cytotoxic T lymphocytes (CTLs), and restrain CD80/CD86 complex via CTLA-4 [42, 44, 45]. Likely, in NK cells, TGF $\beta 1$ can induce overexpression of CD96, and the reductions of CD226 and TIGI $\mathrm{T}$, which break the balance and lead to dysfunction of NK cells in HCC [46]. Meanwhile, TGF- $\beta$ signaling can also enhance PD-1 expression and suppress T-cells function [47]. Taken together, the previous experiments suggested the crucial function of TGF- $\beta$ in immune inhibition.

The networks of TGF- $\beta$ signaling pathway can be approximately divided into two groups. On the one hand, TGF- $\beta$ proteins bind to TGBRs and phosphorylate either SMAD 2 and 3 or SMAD 1, 5, and 8, then they bind to SMAD4 and form a complex [14]. That is the canonical pathway. In HCC, dysregulation of TGF- $\beta$ and SMAD proteins in all kinds of cells is able to cause tumor development and progression. For example, in recent studies, a kind of tumor-inducible, erythroblast-like cells (Tercells) were found gathering in the enlarged spleen, TGF$\beta$ and SMAD3 activation could induce Ter-cells generation, which was important in HCC progression [48]. Furthermore, TGF- $\beta$ can stimulate $\beta$-catenin activation and translocate it into nucleus via SMAD2 and 3 in liver cancer stem cells [49]. On the other hand, noncanonical TGF- $\beta$ signaling pathway, which is SMAD-independent also play crucial roles. In HCC cells, TGF- $\beta$ signaling pathway mediates both pro- and anti-apoptosis process. The anti-apoptosis process is induced by epidermal growth factor receptor (EGFR), which is transactivated by TGF- $\beta$ in the need of TACE/ADAM17 activation [50]. However, Daniel Caballero-Díaz et al. proved the importance of clathrin in induction of proliferative and anti-apoptosis signals by EGFR. In their findings, knockdown of clathrin could not affect phosphorylation of SMAD2, while the suppression of EGFR and AKT phosphorylation was observed. These results indicated that SMAD-independent pathway was involved in transactivation of EGFR [51]. Moreover, TGF- $\beta$ signaling has been found to activate p38/JNK through TGF- $\beta$-activated kinase 1 (TAK1) and Mixed Lineage Kinase 3 (MLK3) in a non-SMAD pathway, holding the balance in cell fate $[52,53]$. Likewise, TGF- $\beta$ activates AKT signals by inducing autocrine regulation of PDGF, which lead to EMT process in HCC [39]. STAT3 activation also participate in stimulating HSCs upon TGF- $\beta$ challenge, which leads to liver fibrosis [54]. In conclusion, TGF- $\beta$ can modulate liver fibrosis and tumor initiation, EMT as well as immune inhibition through both SMAD and SMAD-independent pathways, which plays a dominant role in HCC formation. (Fig. 1).

\section{LncRNAs and the mechanisms of HCC tumorigenesis}

Only $2 \%$ of the genome are able to encode for proteins [55], Non-coding RNAs which are longer than 200 bases are named as long noncoding RNAs (lncRNA) [56]. LncRNAs can be divided into five broad categories: (1) sense and (2) anti-sense which are transcribed from the sense or anti-sense strand of a protein encoding gene; (3) intergenic which locate between two genes; (4) intronic which locate in the whole intron and (5) bidirectional IncRNA when the expression of it and a neighboring coding transcript on the opposite strand locate within $1 \mathrm{~kb}$ from coding transcripts [57, 58]. The mechanisms based on the lncRNAs determine cells fates during their development, and dysregulation of lncRNA may result in many disorders [59]. In HCC, many researches also focus on lncRNAs to find out solutions for earlier diagnosis of liver cancer and effective treatment for advanced stages [60].

It was reported that these lncRNAs were involved in several malignant process of HCC. For example, some lncRNAs were demonstrated to be deregulated in LCSCs and HSCs and could cause the activation of HCC initiation and fibrosis, such as HULC and ANRIL [61, 62]. Likewise, lncRNAs can also participate in tumor growth and metastasis, through regulating EMT, cells proliferation and apoptosis [63-65]. Most of these lncRNAs could serve their functions by regulating the expression of target genes, including protein-coding genes or other non-coding RNAs [66]. Here, we have summarized four different mechanisms of lncRNAs according to their functions targeting DNAs, RNAs and proteins.

Firstly, lncRNAs can modulate gene expression through epigenetic modifications on promoters of target 


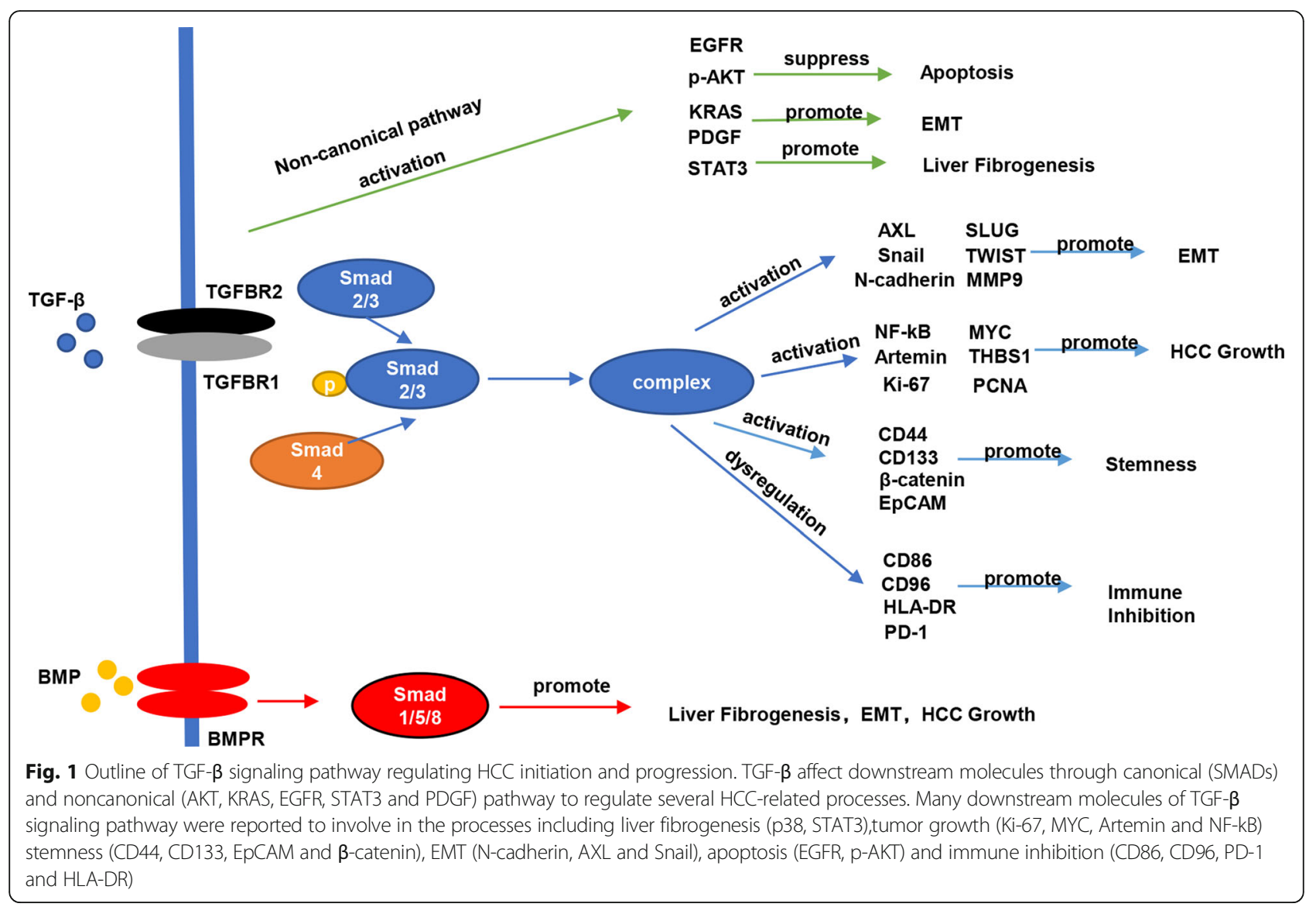

genes. For example, DLX6-AS1 is highly expressed in LCSCs and its overexpression contributes to methylation of cell adhesion molecule 1 (CADM1) promoter, which induce downregulation of CADM1 and activate STAT3 signaling pathway [67]. Likely, in HCC cells, there are similar mechanisms. BZRAP1-AS1 facilitates tumor angiogenesis via inducing methylation of the thrombospondin1 (THBS1) promoter and repressing its transcription [68].Linc-GALH was observed to promote HCC metastasis through controlling the methylation status of Gankyrin, which was associated with ubiquitination of DNA methyltransferases 1 (DNMT1) [69]. Those results have demonstrated the function of lncRNAs in epigenetic regulation by interacting with DNAs.

Secondly, lncRNA can affect alterative splicing (AS) of pre-mRNA, which is important in regulation of gene function. Generally, AS is regulated by trans-acting protein factors [70]. Located in nucleus, some of transacting factors can combine with lncRNAs and lead to diversification of gene expression, which contribute to tumor growth and metastasis in colorectal cancer and breast cancer [71, 72]. Similarly, in HCC, MALAT1 has been found to bind to the splicing factor SRSF1, one of SR proteins, and upregulated the expression of it. Then SRSF1 could regulate AS of S6K1 to activate mTOR pathway, and enhance the production of antiapoptotic splicing isoforms as well [73], indicating that lncRNAs can modulate AS of pre-mRNA by binding to transacting proteins in $\mathrm{HCC}$ development.

Thirdly, some lncRNAs located in cytoplasm may exert their function as competitive endogenous RNA (ceRNA) in HCC development. In ceRNA network, lncRNAs sponge to specific microRNAs and competitively enhance the expression of target messenger RNAs. This mechanism is usually associated with tumor progression and chemotherapy resistance of HCC. For example, HULC was found to sponge to miR-2052 which could target the MET receptor tyrosine kinase. Consequently, overexpression of HULC could stimulate MET, which lead to tumor development and metastasis of HCC [74]. Likewise, EPB41L4A-AS2 was demonstrated to upregulate forkhead box L1 (FOXL1) via binding to miR-301a-5p [75]. The ceRNA network usually exists in malignant process of $\mathrm{HCC}$.

Fourthly, lncRNAs can also bind to proteins, such as transcriptional factor, and modulate their activity and localization, which contribute to HCC progression. Linc00324 could interact with the transcription factor PU box binding protein (PU.1) and recruit it to promote expression of Fas ligand, which is significant to maintain 
the biological properties of LCSCs [76]. Conversely, downregulation of SLC2A1-AS1 in HCC could suppress the combination of signal transducer and activator of transcription 3 (STAT3), which leads to deregulation of FOXM1/GLUT1 axis and contribute to cells proliferation and metastasis [77]. Furthermore, Linc01093 could suppress HCC development by interacting with insulinlike growth factor 2 mRNA-binding protein 1, obstructing interaction between IGF2BP1 and glioma-associated oncogene homolog 1 (GLI1) mRNA [78]. These results showed the function of lncRNAs in HCC by binding to proteins. What's more, some antisense lncRNAs can affect their corresponding mRNA to regulate malignant behavior of HCC. It was reported that LncHOXA10 could bind to SNF2L and recruited NURF chromatin remodeling complex to HOXA10 promoter, then activate HOXA10 in liver tumor initiating cells [79]. Likewise, PCNA-AS1 was observed to promote tumor growth by increasing PCNA mRNA stability [80]. In conclusion, lncRNAs show complicated mechanisms in tumorigenesis and might be possibly therapy targets of $\mathrm{HCC}$ (Table 1).

\section{The interaction of TGF- $\beta$ and IncRNAs in HCC: how they influence each other}

Dysregulation of TGF- $\beta$ and lncRNAs are important inducement in tumorigenesis. During recent decades, researchers have turn to pay more attention to the connection of these two elements, and these findings have revealed that lncRNAs and TGF- $\beta$ could influence each other in several mechanisms of tumor initiation and progression. For example, SMAD2, 3 and 4 could transactivate HOTAIR by binding to its promoter site and promote metastasis of breast cancer [81]. X inactivate-specific transcript (XIST) could facilitate EMT induced by TGF- $\beta$ through miR-367/141-ZEB2 axis in NSCLC [82]. Furthermore, studies of Pachera et al. showed lncRNA H19X, which was served as a critical regulator of TGF- $\beta$-driven tissue fibrosis, could be induced by TGF- $\beta$ in a time- and dose-dependent manners in fibroblasts [83]. Similarly, in HCC TGF- $\beta$ and lncRNAs could be able to influence each other through diverse mechanisms, which have been reported in HCC initiation and progression. In the investigation of Davide Degli Esposti et al., 5525 lncRNAs from different tissues were detected to associate with that in TGF- $\beta$ and 57 of them differentially expressed in HCC compared with adjacent non-tumor tissues, These lncRNAs were coexpressed with genes about liver metabolism and cell cycle [84]. Generally, there are two ways to understand the relation between TGF- $\beta$ and lncRNAs.

For one thing, lncRNAs can be served as upstream molecules of TGF- $\beta$ signaling pathway by activating TGF- $\beta$ and leading to malignant behaviors in HCC. Totally, some lncRNAs have been demonstrated to serve their function through targeting variety of molecules in TGF $\beta$ signaling pathway, including LTBP, TGF $\beta$ s, TGFBR and SMAD proteins. As described earlier, these

Table 1 LncRNAs and their mechanisms in HCC suppression and promotion

\begin{tabular}{llll}
\hline LncRNAs & Effect & Mechanism & Downstream Pathway \\
\hline BZRAP1-AS1 & Oncogene & Epigenetic modification & DNMT3b/THBS1 \\
PVT1 & Oncogene & Epigenetic modification & EZH2/MYC \\
Linc-GALH & Oncogene & Epigenetic modification & CADM1/Stat3 \\
DLX6-AS1 & Tumor suppressor & Epigenetic modification & miR-509-3p/PDGFRA \\
Linc00467 & Oncogene & CeRNA & miR-199a-3p/CDK1,CCNB1 \\
Linc00346 & Oncogene & CeRNA & miR-675/PKM2 \\
HULC & Oncogene & CeRNA & miR-2052/MET \\
RP11-422 N16.3 & & CeRNA & miR-23b-3p/DMGDH \\
MALAT1 & Tumor suppressor & CeRNA & miR-140NEGFA \\
HAND2-AS1 & Oncogene & CeRNA & SRSF1/S6K1 \\
Linc00324 & & Alterative Splicing & INO80/BMPR1A \\
Linc01093 & Binding to proteins & PU.1/FASL \\
SLC2A1-AS1 & Oncogene & Binding to proteins & IGF2BP1/GLI1 \\
PCNA1-AS1 & Oncogene & Binding to proteins & STAT3/FOXM1/GLUT1 \\
LnCHOXA10 & Tumor suppressor & Binding to proteins & PCNA1
\end{tabular}

BZRAP1-AS1 benzodiazapine receptor associated protein 1 antisense RNA 1; DNMT3b DNA methyltransferase 3B; THBS1 thrombospondin-1; PVT1 plasmacytoma variant translocation 1; HULC highly upregulated in liver cancer; Malat1, metastasis-associated lung adenocarcinoma transcript 1; HAND2 heart and neural crest derivatives-expressed transcript 2; DMGDH Dimethylglycine dehydrogenase; GLUT1 Glucose transporter type 1; FOXM1 Forkhead box protein M1 
lncRNA affect TGF- $\beta$ signaling pathway via similar mechanisms. Firstly, lncRNAs can modulate TGF- $\beta$ / SMAD pathway through epigenetic modifications. For example, Lnc34a was recently indicated to interact with epigenetic regulators and methylate the miR-34a promoter to downregulate miR-34a expression, while miR34 a could target SMAD4 in TGF- $\beta$ signaling pathway. Consequently, these findings revealed that Lnc34a could promote bone metastasis of HCC [85]. Secondly, lncRNAs can act as ceRNA to regulate TGF- $\beta$. NOARD is a lncRNA upregulated in HCC and correlated with the poor prognosis. In previous studies, NOARD was observed to competitively interact with miR-202-5p, which targets TGFBR1 and TGFBR2 [86]. HANR and SBF2-AS1 also exerted their functions through sponging to microRNAs which target TGFBRs $[87,88]$. Meanwhile, Bai et al. showed that A1BG-AS1 could positively regulate SMAD7 and suppress HCC cells proliferation and invasion by sponging to miR-246a-5p [89]. Moreover, lncRNAs can directly interact with proteins to modulate TGF- $\beta$ signaling pathway. For example, LncLFAR1 could directly bind to SMAD2 and 3, and then enhance the expression of SMADs to promote their phosphorylation in liver fibrogenesis [90]. LncRNAs can also regulate BMP signaling through this mechanism. HAND2-AS1 was found to bind to INO80 and recruit the chromatin-remodeling complex to the promoter of BMPR1A and activate BMP signaling [91]. Dysregulation of these IncRNA can cause proliferation and metastasis of HCC cells and facilitate tumor initiation and progression (Fig. 2).

Furthermore, malignant functions of some lncRNAs can also be induced by TGF- $\beta$ in HCC. LncATB is the most classical target lncRNAs of TGF- $\beta$ signaling, named LncRNA activated by TGF- $\beta$. LncATB was firstly found to be upregulated in $\mathrm{HCC}$ and associated with poor prognosis $[92,93]$. In HCC, LncATB could competitively bind to miR-200 family and consequently promote ZEB1 and ZEB2, which could lead to EMT and tumor metastasis $[92,94]$. In another example, lncATB

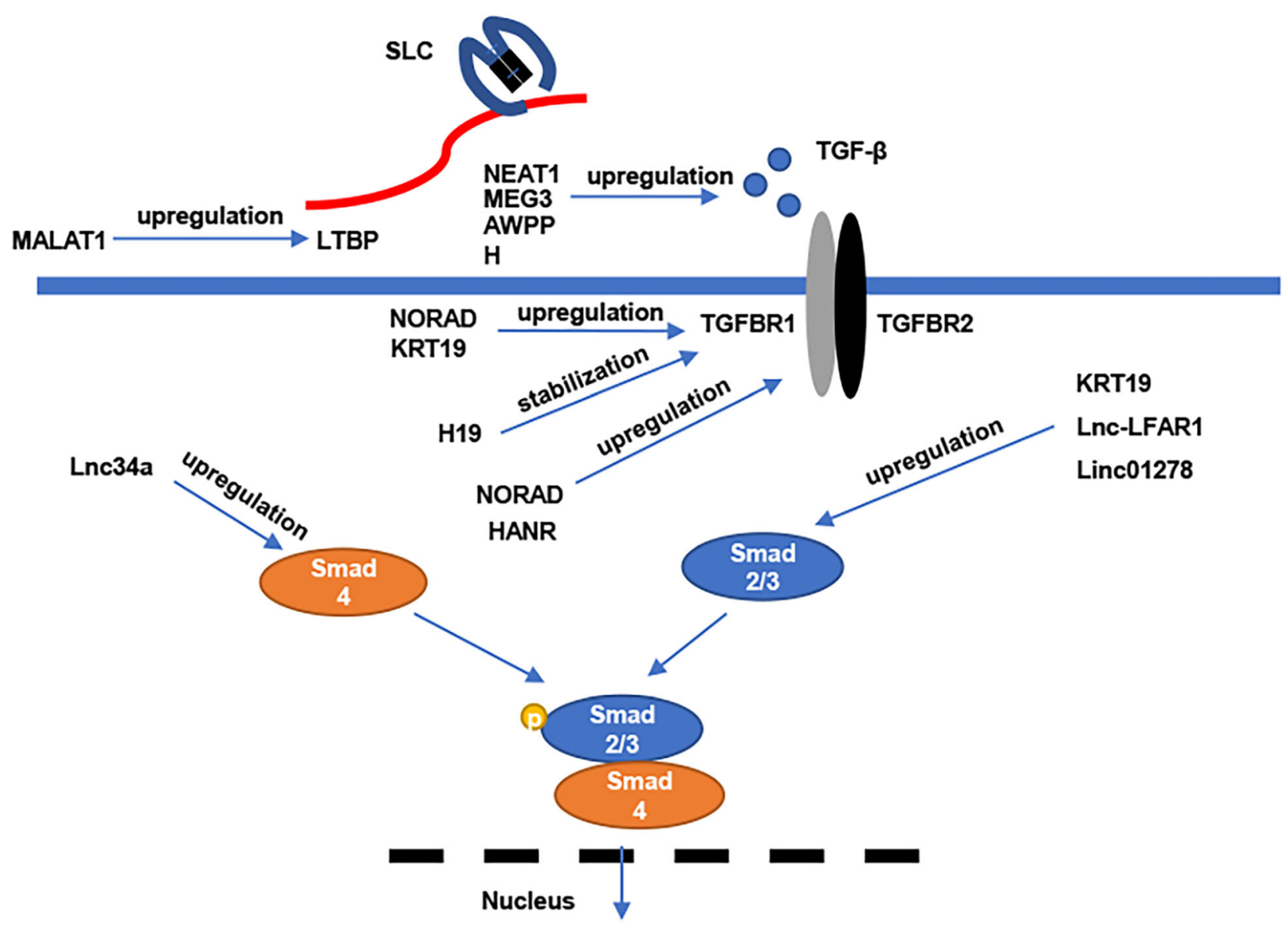

Fig. 2 Function of InCRNAs in regulating TGF- $\beta$ signaling pathway to affect HCC tumorigenesis. In HCC, regulation of many related or downstream molecules in TGF- $\beta$ signaling pathway were reported to be relevant to specific IncRNAs: upregulation of LTBP (MALAT1), upregulation of TGF $\beta 1$ (NEAT1, MEG3 and AWPPH), upregulation of SMADs (LnC34a, KRT19, Lnc-LFAR1 and LINC01278), upregulation or stabilization of TGFBRs (NORAD, KRT19, HANR and H19). These IncRNAs affect TGF- $\beta$ signaling pathway by many action sites of downstream molecules 
could also bind to autophagy-related protein 5 mRNA and promote its expression, in order to activate HippoYAP signaling, in order to promote autophagy [95]. Meanwhile, there are many other lncRNA targeted by TGF- $\beta$. UCA1 could be upregulated by TGF $\beta 1$ and promote tumor growth of HCC via influencing lactate production, glucose uptake and ATP production [96]. Liang et al. showed the overexpression of SLC7A11-AS1 by induction of TGF- $\beta$ could facilitate HCC metastasis [97]. Moreover, LncRNA H19 expression was reduced by TGF- $\beta$ through Sox 2 and increased in TGFBR knocked out tumor-initiating hepatocytes (TICs). TUNEL assays showed that H19 knockdown caused apoptosis of TICs through TGF- $\beta$, and H19 is important in TICs progenitor capacity [98]. Taken together, these findings demonstrated that lncRNAs could be downstream molecules of TGF- $\beta$ signaling to exert their functions in HCC initiation and progression.

Besides, there is a discovery of mechanism in LncRNA-hPVT1. In WANG et al. investigation, two independent lncRNAs microarray showed that LncRNAhPVT1 could activate TGF- $\beta$ signaling pathway. Simultaneously, LncRNA-hPVT1 could be induced by TGF- $\beta$. That means a feedback loop between these two elements in HCC progression [99] (Table 2).

\section{The possible therapeutic targets of HCC revealed in studies of TGF $\beta$ and IncRNAs}

$\mathrm{HCC}$ is the most common type of primary liver cancer. The tumorigenesis of HCC is a complicated multi-step process containing multiple molecules and multiple signaling pathways. Therefore, investigation of molecule mechanisms in HCC initiation and progression might help us improve therapy strategies for HCC [100]. For example, recent studies revealed a novel molecule inhibitor of TGFBR1 named galunisertib. In phase 2 clinical study of galunisertib in HCC patients and Child-Pugh A $5 / 6$ or B7, the results showed that galunisertib could decrease level of AFP and prolong overall survival and progression-free survival [101]. And another TGFBR1 inhibitor LY2109761 has been observed to improve curative effect of TAE and suppressing metastasis [102]. In addition to the canonical pathway via TGFBR and SMAD proteins, noncanonical pathways such as $\mathrm{p}-\mathrm{AKT}$ activated by TGF- $\beta$ may be useful targets [103]. Moreover, as TGF- $\beta$ signaling pathway suppress immune response, a dual-targeting inhibitor of TGF- $\beta$ and PD-L1 was reported. It might enhance cytolytic ability of NK cells and reduce suppressive activity of Treg cells [104].

Combination treatment is now with the increasing appreciation in HCC because it has been shown to possess more sensitivity and less tolerance resistance than those in single drug. For example, an anti-malaria drug named artesunate was reported to collaborate with sorafenib and improve its efficacy in HCC through targeting ERK and STAT3, while combination with galunisertib could enhance the efficacy of sorafenib by delaying drug resistance of tumor $[105,106]$. In our framework, we summarize the lncRNAs involved in TGF- $\beta$ signaling pathway and provide possibility of collaboration between TGF- $\beta$ inhibitors and lncRNAs or their downstream molecules in treatment of HCC. Firstly, several lncRNAs related to TGF- $\beta$ have been found to influence chemotherapeutic resistance in HCC. For example, HOTAIR was reported to be upregulated by TGF $\beta 1$ and involved in TGF $\beta 1$-induced multidrug resistance (MDR) [107]. In Takahashi et al. studies, similar results happened on Linc-ROR, indicated that extracellular vesicles located Linc-ROR contribute to chemoresistance [108]. These lncRNAs could be directly targeted to enhance curative effect in combined pharmacotherapy. Secondly, some lncRNAs mentioned in TGF- $\beta$ signaling pathway show relevant mechanisms with TGF- $\beta$ proteins, which make it possible for synergistic anticancer effect in process of HCC tumorigenesis and progression. In another example, as an important mediate molecule in TGF- $\beta$ signaling pathway, LncATB was demonstrated to competitively upregulate ZEB and trigger STAT3 signaling [92, 94]. Therefore, in our hypothesize, silencing of LncATB could suppress the ZEB and STAT3 signaling

Table 2 Downstream IncRNAs of TGF- $\beta$ signaling in HCC

\begin{tabular}{llll}
\hline LnCRNAs & Regulation by TGF- $\boldsymbol{\beta}$ & Downstream molecules & Function in HCC \\
\hline LnCATB & Upregulation & miR-200/ZEB1, ZEB2 & EMT \\
& & YAP, ATG5 & Autophagy \\
HOTAIR & Upregulation & miR-145/P-gp, BCRP & Multidrug resistance \\
HCCL5 & Upregulation & N-cadherin, E-cadherin & EMT \\
UCA1 & Upregulation & HXK2 & Tumor Growth \\
Linc-ROR & Upregulation & Caspase 8, GADD45B & Chemoresistance \\
PVT1 & Upregulation & NOP2 & Tumor Growth \\
H19 & Downregulation & TP53/CDKI1A & Progenitor Capacity \\
\hline
\end{tabular}

TGF- $\beta$ transforming growth factor- $\beta$; ATG5 Autophagy Related 5; HOTAIR HOX transcript antisense RNA; UCA1 urothelial carcinoma antigen 1; HXK2 Hexokinase-2; GADD45B Growth arrest and DNA damage-inducible protein GADD45 beta; PVT1 plasmacytoma variant translocation 1; NOP2 Nucleolar Protein 2 
induced by TGF- $\beta$ and might enhance the anticancer effect of TGF- $\beta$ inhibitor. Moreover, it is worthy to notice that IncRNA H19 could be both upstream and downstream molecule of TGF- $\beta$ [98, 109]. These findings might reveal multiple synergistic effect between TGF- $\beta$ and H19. Thirdly, although there are also amounts of lncRNAs which are independent to TGF- $\beta$ signaling pathway. Some of these lncRNAs are able to induce same downstream molecules with TGF- $\beta$, makes it possible for cooperation in HCC treatment. For instance, the TGFBR1 inhibitor galunisertib can block VEGF synthesis [110], while MALAT1 was observed to enhance VEGF production via inhibiting miR-140 and promoted angiogenesis of HUVECs in HCC [111]. These results showed possible combination between galunisertib and MALAT1 inhibitors in HCC treatment targeting angiogenesis. In addition, the TGF- $\beta$ inhibitor SB431542 and CASC2c could repress ERK signaling and inhibit cell proliferation in $\mathrm{HCC}$, which provide a novel therapeutic target for HCC $[65,112]$. Taken together, by analyzing these findings, we hypothesize that many novel therapeutic strategies of combined pharmacotherapy for HCC could be found in collaboration between TGF- $\beta$ and lncRNAs.

\section{Conclusion}

Hepatocellular carcinoma is still a great menace for human-beings, and both therapy and early diagnosis methods need to be perfected. In our review, we paid considerable attention to lncRNAs and TGF- $\beta$ signaling pathway, which have been studied in HCC for years. Totally, TGF- $\beta$ has been proved to play distinct roles in HCC tumorigenesis through both canonical and noncanonical pathway. EMT is the major effect under induction of TGF- $\beta$ signaling. Moreover, in tumor initiation and immune inhibition, TGF- $\beta$ also serves as an important promoter. Other members of TGF- $\beta$ superfamily like BMPs involve in HCC tumorigenesis as well.

When lncRNAs participate in dysregulation of TGF- $\beta$ signaling pathway in HCC, they can exert their functions in varieties of different mechanisms. According to the subcellular location of these lncRNAs, they can affect DNA, RNA and proteins through epigenetic modification, alterative splicing, competitively sponge and directly binding. LncRNAs have been demonstrated to affect most of steps in TGF- $\beta$ signaling pathway, such as TGF- $\beta$ proteins, receptor and downstream SMAD2, 3 and 4 . However, there are also many lncRNAs regulated by TGF- $\beta$. In this review, we have summarized several typical downstream lncRNAs of TGF- $\beta$ signaling. These results are important complements of mechanisms in $\mathrm{HCC}$ initiation and progression.

In addition, investigations about dysregulation of TGF- $\beta$ signaling pathway and lncRNAs may provide some promising therapeutic targets for HCC. Besides single drugs targeting TGF- $\beta$ or some typical lncRNAs, we elucidate the possibility of combined pharmacotherapy by simultaneously targeting TGF- $\beta$ signaling and special lncRNAs which could influence TGF- $\beta$ signaling or be affected by the same downstream pathway of TGF- $\beta$. All in all, TGF- $\beta$ and lncRNAs are promising therapeutic targets, and may provide some novel ideas about combined pharmacotherapy in HCC.

\begin{abstract}
Abbreviations
HCC: Hepatocellular carcinoma; TGF- $\beta$ : Transforming growth factor- $\beta$; BMP: Bone morphogenic protein; LAP: Latency-associated peptide; SLC: Small latent complex; LTBP: Latent TGF- $\beta$ binding protein; HPC: Hepatic progenitor cell; CTGF: Connective tissue growth factor; LCSC: Liver cancer stem cell; HSC: Hepatic stellate cell; TAM: Tumor-associated macrophage; TAN: Tumorassociated neutrophil; PDGF: Platelet-derived growth factor; EMT: Epithelialmesenchymal transition; PD-1: Programmed cell death protein 1; EGFR: Epidermal growth factor receptor; STAT3: Signal transducer and activator of transcription 3; DNMT1: DNA methyltransferase 1;

ERK: Extracellular regulated MAP kinase; AS: Alterative splicing; snRNP: Small nuclear ribonucleoprotein; MALAT1: Metastasis associated lung adenocarcinoma transcript 1; HULC: Hepatocellular carcinoma up-regulated long non-coding RNA; LncATB: Long noncoding RNA activated by TGF- $\beta$
\end{abstract}

\section{Acknowledgements}

Not applicable.

Authors' contributions

XP Chen and BX Zhang conceived this study. MZ Han, ZB Liao and FR Liu drafted the manuscript. All authors read and approved the final manuscript.

\section{Funding}

This work was supported by grants from National Natural Science Foundation of China $(81572855,81874189)$.

Availability of data and materials

Not applicable.

Ethics approval and consent to participate

Not applicable.

Consent for publication

Not applicable.

Competing interests

The authors declare that they have no competing interests.

\section{Author details}

${ }^{1}$ Hepatic Surgery Center, Tongji Hospital, Tongji Medical College, Huazhong University of Science and Technology, 1095 Jiefang Avenue, Wuhan 430030, China. ${ }^{2}$ Hubei Key Laboratory of Hepato-Pancreato-Biliary Diseases, Wuhan 430030, China.

Received: 18 September 2020 Accepted: 24 November 2020

Published online: 01 December 2020

References

1. Peng R, Zhang P, Yang X, Wei C, Huang X, Cai J, et al. Overexpression of RNF38 facilitates TGF- $\beta$ signaling by Ubiquitinating and degrading AHNAK in hepatocellular carcinoma. J Exp Clin Canc Res. 2019;38.

2. Wang M, Zhang L, Liu Z, Zhou J, Pan Q, Fan J, et al. AGO1 may influence the prognosis of hepatocellular carcinoma through TGF- $\beta$ pathway. Cell Death Dis. 2018;9.

3. Lu C, Rong D, Zhang B, Zheng W, Wang X, Chen Z, et al. Current perspectives on the immunosuppressive tumor microenvironment in hepatocellular carcinoma: challenges and opportunities. Mol Cancer. 2019;18. 
4. Lan T, Li H, Zhang D, Xu L, Liu H, Hao X, et al. KIAA1429 contributes to liver cancer progression through N6-methyladenosine-dependent posttranscriptional modification of GATA3. Mol Cancer. 2019;18.

5. Wang $H$, Lin X, Liu E, Jian Z, Ou Y. MicroRNA-33b regulates hepatocellular carcinoma cell proliferation, apoptosis, and mobility via targeting Fli-1mediated Notch1 pathway. J Cell Physiol. 2020.

6. Ahmadi A, Najafi M, Farhood B, Mortezaee K. Transforming growth factor- $\beta$ signaling: tumorigenesis and targeting for cancer therapy. J Cell Physiol. 2018;234:12173-87.

7. Padua D, Massague J. Roles of TGF beta in metastasis. Cell Res. 2009;19:89-102.

8. Katz LH, Likhter M, Jogunoori W, Belkin M, Ohshiro K, Mishra L. TGF- $\beta$ signaling in liver and gastrointestinal cancers. Cancer Lett. 2016;379:166-72.

9. Yu Y, Feng X. TGF- $\beta$ signaling in cell fate control and cancer. Curr Opin Cell Biol. 2019;61:56-63.

10. Huang Z, Zhou J, Peng Y, He W, Huang C. The role of long noncoding RNAs in hepatocellular carcinoma. Mol Cancer. 2020;19.

11. Connerty P, Lock RB, de Bock CE. Long non-coding RNAs: major regulators of cell stress in Cancer. Front Oncol. 2020;10.

12. Hayashi H, Sakai T. Biological significance of local TGF- $\beta$ activation in liver diseases. Front Physiol. 2012;3.

13. Boguslawska J, Kryst P, Poletajew S, Piekielko-Witkowska A. TGF- $\beta$ and microRNA interplay in genitourinary cancers. Cells-Basel. 2019;8:1619.

14. Massague J. TGFbeta in Cancer Cell. 2008;134:215-30

15. Shi Y, Massague J. Mechanisms of TGF-beta signaling from cell membrane to the nucleus. Cell. 2003;113:685-700.

16. Dewidar B, Meyer C, Dooley S, Meindl-Beinker AN. TGF-beta in hepatic stellate cell activation and liver Fibrogenesis-updated 2019. Cells-Basel. 2019;8.

17. Zhang L, Zhou F, Ten Dijke P. Signaling interplay between transforming growth factor $\beta$ receptor and PI3K/AKT pathways in cancer. Trends Biochem Sci. 2013;38:612-20.

18. Lee MK, Pardoux C, Hall MC, Lee PS, Warburton D, Qing J, et al. TGF-beta activates Erk MAP kinase signalling through direct phosphorylation of ShcA. EMBO J. 2007;26:3957-67.

19. Hao Y, Baker D, Ten Dijke P. TGF- $\beta$-mediated epithelial-Mesenchymal transition and Cancer metastasis. Int J Mol Sci. 2019;20:2767.

20. Meulmeester $E$, Ten Dijke P. The dynamic roles of TGF- $\beta$ in cancer. J Pathol. 2011;223:206-19

21. Sorrentino A, Thakur N, Grimsby S, Marcusson A, von Bulow V, Schuster N, et al. The type I TGF-beta receptor engages TRAF6 to activate TAK1 in a receptor kinase-independent manner. Nat Cell Biol. 2008:10:1199-207.

22. Itatani Y, Kawada K, Sakai Y. Transforming growth factor- $\beta$ signaling pathway in colorectal Cancer and its tumor microenvironment. Int J Mol Sci. 2019:20:5822.

23. Rao S, Mishra L. Targeting transforming growth factor Beta signaling in liver Cancer. Hepatology. 2019;69:1375-8.

24. Fabregat I, Caballero-Díaz D. Transforming growth factor- $\beta$-induced cell plasticity in liver fibrosis and Hepatocarcinogenesis. Front Oncol. 2018;8.

25. Yamazaki K, Masugi Y, Sakamoto M. Molecular pathogenesis of hepatocellular carcinoma: altering transforming growth factor- $\beta$ signaling in Hepatocarcinogenesis. Dig Dis. 2011;29:284-8.

26. Wu Y, Ding Z, Jin $G$, Xiong $Y, Y u B$, Sun $Y$, et al. Autocrine transforming growth factor-B/activin A-Smad signaling induces hepatic progenitor cells undergoing partial epithelial-mesenchymal transition states. Biochimie. 2018;148:87-98.

27. Ding ZY, Jin GN, Liang HF, Wang W, Chen WX, Datta PK, et al. Transforming growth factor beta induces expression of connective tissue growth factor in hepatic progenitor cells through Smad independent signaling. Cell Signal. 2013;25:1981-92

28. Liu YC, Yeh CT, Lin KH. Cancer stem cell functions in hepatocellular carcinoma and comprehensive therapeutic strategies. Cells-Basel. 2020;9.

29. Fan QM, Jing YY, Yu GF, Kou XR, Ye F, Gao L, et al. Tumor-associated macrophages promote cancer stem cell-like properties via transforming growth factor-beta1-induced epithelial-mesenchymal transition in hepatocellular carcinoma. Cancer Lett. 2014;352:160-8.

30. Zhou SL, Yin D, Hu ZQ, Luo CB, Zhou ZJ, Xin HY, et al. A positive feedback loop between Cancer stem-like cells and tumor-associated neutrophils controls hepatocellular carcinoma progression. Hepatology. 2019;70:1214-30.

31. Yoshida GJ, Azuma A, Miura Y, Orimo A. Activated fibroblast program orchestrates tumor initiation and progression; molecular mechanisms and the associated therapeutic strategies. Int J Mol Sci. 2019:20.

32. Ding $N$, Hah N, Yu RT, Sherman MH, Benner C, Leblanc M, et al. BRD4 is a novel therapeutic target for liver fibrosis. Proc Natl Acad Sci U S A. 2015;112: 15713-8.
33. Yoshida GJ. Emerging role of epithelial-mesenchymal transition in hepatic cancer. J Exp Clin Cancer Res. 2016:35:141.

34. Chen J, Zhu H, Liu Q, Ning D, Zhang Z, Zhang L, et al. DEPTOR induces a partial epithelial-to-mesenchymal transition and metastasis via autocrine TGF $\beta 1$ signaling and is associated with poor prognosis in hepatocellular carcinoma. J Exp Clin Canc Res. 2019;38.

35. Qu Z, Feng J, Pan H, Jiang Y, Duan Y, Fa Z. Exosomes derived from HCC cells with different invasion characteristics mediated EMT through TGF-beta/ Smad signaling pathway. Onco Targets Ther. 2019;12:6897-905.

36. Giannelli G, Koudelkova P, Dituri F, Mikulits W. Role of epithelial to mesenchymal transition in hepatocellular carcinoma. J Hepatol. 2016;65: 798-808.

37. Xie $B$, Lin $W$, Ye J, Wang $X$, Zhang $B$, Xiong $S$, et al. DDR2 facilitates hepatocellular carcinoma invasion and metastasis via activating ERK signaling and stabilizing SNAIL1. J Exp Clin Cancer Res. 2015:34:101.

38. Reichl P, Dengler M, van Zijl F, Huber H, Fuhrlinger G, Reichel C, et al. Axl activates autocrine transforming growth factor-beta signaling in hepatocellular carcinoma. Hepatology. 2015;61:930-41.

39. Fischer AN, Fuchs E, Mikula M, Huber H, Beug H, Mikulits W. PDGF essentially links TGF-beta signaling to nuclear beta-catenin accumulation in hepatocellular carcinoma progression. Oncogene. 2007;26:3395-405.

40. Liao Z, Chen L, Zhang X, Zhang H, Tan X, Dong K, et al. PTPRE acts as a metastatic promoter in hepatocellular carcinoma by facilitating recruitment of SMAD3 to TGF- $\beta$ receptor 1. Hepatology. 2020.

41. Wu M, Yiang G, Cheng P, Chu P, Li C. Molecular targets in Hepatocarcinogenesis and implications for therapy. J Clin Med. 2018;7:213.

42. Chen J, Gingold JA, Su X. Immunomodulatory TGF- $\beta$ signaling in hepatocellular carcinoma. Trends Mol Med. 2019;25:1010-23.

43. Ringelhan M, Pfister D. O Connor T, Pikarsky E, Heikenwalder M. the immunology of hepatocellular carcinoma. Nat Immunol. 2018;19:222-32.

44. Cariani E, Missale G. Immune landscape of hepatocellular carcinoma microenvironment: implications for prognosis and therapeutic applications. Liver Int. 2019;39:1608-21.

45. Li MO, Flavell RA. TGF-B: a master of all T cell trades. Cell. 2008:134:392-404.

46. Sun $H$, Huang $Q$, Huang $M$, Wen $H$, Lin $R$, Zheng $M$, et al. Human CD96 correlates to natural killer cell exhaustion and predicts the prognosis of human hepatocellular carcinoma. Hepatology. 2019;70:168-83.

47. Park BV, Freeman ZT, Ghasemzadeh A, Chattergoon MA, Rutebemberwa A, Steigner J, et al. TGF 1-mediated SMAD3 enhances PD-1 expression on antigen-specific T cells in Cancer. Cancer Discov. 2016;6:1366-81.

48. Han Y, Liu Q, Hou J, Gu Y, Zhang Y, Chen Z, et al. Tumor-induced generation of splenic erythroblast-like Ter-cells promotes tumor progression. Cell. 2018;173:634-48.

49. Chen Y, Meng L, Shang H, Dou Q, Lu Z, Liu L, et al. $\beta 2$ spectrin-mediated differentiation repressed the properties of liver cancer stem cells through $\beta$ catenin. Cell Death Dis. 2018;9.

50. Murillo MM, Del CG, Sanchez A, Fernandez M, Fabregat I. Involvement of EGF receptor and c-Src in the survival signals induced by TGF-beta1 in hepatocytes. Oncogene. 2005;24:4580-7.

51. Caballero-Díaz D, Bertran E, Peñuelas-Haro I, Moreno-Càceres J, Malfettone A, López-Luque J, et al. Clathrin switches transforming growth factor- $\beta$ role to pro-tumorigenic in liver cancer. J Hepatol. 2020;72:125-34.

52. Kim KY, Kim BC, Xu Z, Kim SJ. Mixed lineage kinase 3 (MLK3)-activated p38 MAP kinase mediates transforming growth factor-beta-induced apoptosis in hepatoma cells. J Biol Chem. 2004:279:29478-84.

53. Yang L, Inokuchi S, Roh YS, Song J, Loomba R, Park EJ, et al. Transforming growth factor- $\beta$ signaling in hepatocytes promotes hepatic fibrosis and carcinogenesis in mice with hepatocyte-specific deletion of TAK1. Gastroenterology. 2013;144:1042-54.

54. Liu Y, Liu H, Meyer C, Li J, Nadalin S, Königsrainer A, et al. Transforming growth factor- $\beta$ (TGF- $\beta$ )-mediated connective tissue growth factor (CTGF) expression in hepatic stellate cells requires Stat3 signaling activation. J Biol Chem. 2013;288:30708-19.

55. Djebali S, Davis CA, Merkel A, Dobin A, Lassmann T, Mortazavi A, et al. Landscape of transcription in human cells. Nature. 2012;489:101-8.

56. Nagano T, Fraser P. No-nonsense functions for long noncoding RNAs. Cell. 2011;145:178-81.

57. Huo X, Han S, Wu G, Latchoumanin O, Zhou G, Hebbard L, et al. Dysregulated long noncoding RNAs (IncRNAs) in hepatocellular carcinoma: implications for tumorigenesis, disease progression, and liver cancer stem cells. Mol Cancer. 2017;16. 
58. Ponting CP, Oliver PL, Reik W. Evolution and functions of long noncoding RNAs. Cell. 2009;136:629-41.

59. Batista PJ, Chang HY. Long noncoding RNAs: cellular address codes in development and disease. Cell. 2013;152:1298-307.

60. Klingenberg M, Matsuda A, Diederichs S, Patel T. Non-coding RNA in hepatocellular carcinoma: mechanisms, biomarkers and therapeutic targets. J Hepatol. 2017:67:603-18.

61. Yang JJ, Yang Y, Zhang C, Li J, Yang Y. Epigenetic silencing of LnCRNA ANRI L enhances liver fibrosis and HSC activation through activating AMPK pathway. J Cell Mol Med. 2020;24:2677-87.

62. Wang $C$, Jiang X, Li X, Song S, Meng Q, Wang L, et al. Long noncoding RNA HULC accelerates the growth of human liver cancer stem cells by upregulating CyclinD1 through miR675-PKM2 pathway via autophagy. Stem Cell Res Ther. 2020;11:8.

63. Sun Y, Zhou Q, Li J, Zhao C, Yu Z, Zhu Q. LncRNA RP11-422N16.3 inhibits cell proliferation and EMT, and induces apoptosis in hepatocellular carcinoma cells by sponging miR-23b-3p. Onco Targets Ther. 2019;12: 10943-61.

64. Li J, Guo W, Xue W, Xu P, Deng Z, Zhang D, et al. Long noncoding RNA AURKAPS1 potentiates malignant hepatocellular carcinoma progression by regulating miR-142, miR-155 and miR-182. Sci Rep-Uk. 2019;9.

65. Li QY, Yang K, Liu FG, Sun XG, Chen L, Xiu H, et al. Long noncoding RNA CASC2c inhibited cell proliferation in hepatocellular carcinoma by inactivated ERK1/2 and Wnt/ß-catenin signaling pathway. Clin Transl Oncol. 2020;22:302-10.

66. Parasramka MA, Maji S, Matsuda A, Yan IK, Patel T. Long non-coding RNAs as novel targets for therapy in hepatocellular carcinoma. Pharmacol Therapeut. 2016;161:67-78.

67. Wu D, Zheng Z, Zhang Y, Fan S, Zhang Z, Wang Y, et al. Down-regulated IncRNA DLX6-AS1 inhibits tumorigenesis through STAT3 signaling pathway by suppressing CADM1 promoter methylation in liver cancer stem cells. J Exp Clin Canc Res. 2019;38.

68. Wang W, Chen G, Wang B, Yuan Z, Liu G, Niu B, et al. Long non-coding RNA BZRAP1-AS1 silencing suppresses tumor angiogenesis in hepatocellular carcinoma by mediating THBS1 methylation. J Transl Med. 2019;17.

69. Xu X, Lou Y, Tang J, Teng Y, Zhang Z, Yin Y, et al. The long non-coding RNA Linc-GALH promotes hepatocellular carcinoma metastasis via epigenetically regulating Gankyrin. Cell Death Dis. 2019;10.

70. Tripathi V, Ellis JD, Shen Z, Song DY, Pan Q, Watt AT, et al. The nuclearretained noncoding RNA MALAT1 regulates alternative splicing by modulating SR splicing factor phosphorylation. Mol Cell. 2010;39:925-38.

71. Huan L, Guo T, Wu Y, Xu L, Huang S, Xu Y, et al. Hypoxia induced LUCAT1/ PTBP1 axis modulates cancer cell viability and chemotherapy response. Mol Cancer. 2020;19.

72. De Troyer L, Zhao P, Pastor T, Baietti MF, Barra J, Vendramin R, et al. Stressinduced IncRNA LASTR fosters cancer cell fitness by regulating the activity of the U4/U6 recycling factor SART3. Nucleic Acids Res. 2020;48:2502-17.

73. Malakar P, Shilo A, Mogilevsky A, Stein I, Pikarsky E, Nevo Y, et al. Long noncoding RNA MALAT1 promotes hepatocellular carcinoma development by SRSF1 Upregulation and mTOR activation. Cancer Res. 2017;77:1155-67.

74. Zhang H, Liao Z, Liu F, Su C, Zhu H, Li Y, et al. Long noncoding RNA HULC promotes hepatocellular carcinoma progression. Aging. 2019;11:9111-27.

75. Wang Y, Wang T, Shi M, Zhai B. Long noncoding RNA EPB41L4A-AS2 inhibits hepatocellular carcinoma development by sponging miR-301a-5p and targeting FOXL1. J Exp Clin Canc Res. 2019;38:1.

76. Gao J, Dai C, Yu X, Yin XB, Zhou F. Long noncoding RNA LINC00324 exerts protumorigenic effects on liver cancer stem cells by upregulating fas ligand via PU box binding protein. FASEB J. 2020;34:5800-17.

77. Shang R, Wang M, Dai B, Du J, Wang J, Liu Z, et al. Long noncoding RNA SLC2A1-AS1 regulates aerobic glycolysis and progression in hepatocellular carcinoma via inhibiting the STAT3/FOXM1/GLUT1 pathway. Mol Oncol. 2020;14:1381.

78. He J, Zuo Q, Hu B, Jin H, Wang C, Cheng Z, et al. A novel, liver-specific long noncoding RNA LINC01093 suppresses HCC progression by interaction with IGF2BP1 to facilitate decay of GLI1 mRNA. Cancer Lett. 2019;450:98-109.

79. Shao M, Yang Q, Zhu W, Jin H, Wang J, Song J, et al. LncHOXA10 drives liver TICs self-renewal and tumorigenesis via HOXA10 transcription activation. Mol Cancer. 2018;17:1.

80. Yuan S, Tao Q, Wang J, Yang F, Liu L, Wang L, et al. Antisense long non-coding RNA PCNA-AS1 promotes tumor growth by regulating proliferating cell nuclear antigen in hepatocellular carcinoma. Cancer Lett. 2014;349:87-94.
81. Ren Y, Jia H, Xu Y, Zhou X, Zhao X, Wang Y, et al. Paracrine and epigenetic control of CAF-induced metastasis: the role of HOTAIR stimulated by TGF-B1 secretion. Mol Cancer. 2018;17.

82. Li C, Wan L, Liu Z, Xu G, Wang S, Su Z, et al. Long non-coding RNA XIST promotes TGF- $\beta$-induced epithelial-mesenchymal transition by regulating miR367/141-ZEB2 axis in non-small-cell lung cancer. Cancer Lett. 2018;418:185-95.

83. Pachera E, Assassi S, Salazar GA, Stellato M, Renoux F, Wunderlin A, et al. Long noncoding RNA H19X is a key mediator of TGF-beta-driven fibrosis. J Clin Invest. 2020;130:4888-905.

84. Esposti DD, Hernandez-Vargas H, Voegele C, Fernandez-Jimenez N, Forey N, Bancel $B$, et al. Identification of novel long non-coding RNAs deregulated in hepatocellular carcinoma using RNA-sequencing. Oncotarget. 2016;7:31862.

85. Zhang L, Niu H, Ma J, Yuan B, Chen Y, Zhuang Y, et al. The molecular mechanism of LncRNA34a-mediated regulation of bone metastasis in hepatocellular carcinoma. Mol Cancer. 2019:18:120.

86. Yang X, Cai JB, Peng R, Wei CY, Lu JC, Gao C, et al. The long noncoding RNA NORAD enhances the TGF- $\beta$ pathway to promote hepatocellular carcinoma progression by targeting miR-202-5p. J Cell Physiol. 2018;234: 12051-60.

87. Shi $Y$, Yang $X$, Xue $X$, Sun $D$, Cai $P$, Song $Q$, et al. HANR promotes hepatocellular carcinoma progression via miR-214/EZH2/TGF- $\beta$ axis. Biochem Bioph Res Co. 2018;506:189-93.

88. Li Y, Liu G, Li X, Dong H, Xiao W, Lu S. Long non-coding RNA SBF2-AS1 promotes hepatocellular carcinoma progression through regulation of miR140-5p-TGFBR1 pathway. Biochem Bioph Res Co. 2018;503:2826-32.

89. Bai J, Yao B, Wang L, Sun L, Chen T, Liu R, et al. IncRNA A1BG-AS1 suppresses proliferation and invasion of hepatocellular carcinoma cells by targeting miR-216a-5p. J Cell Biochem. 2019;120:10310-22.

90. Zhang K, Han X, Zhang Z, Zheng L, Hu Z, Yao Q, et al. The liver-enriched Inc-LFAR1 promotes liver fibrosis by activating TGF $\beta$ and notch pathways. Nat Commun. 2017:8:1.

91. Wang Y, Zhu P, Luo J, Wang J, Liu Z, Wu W, et al. LncRNA HAND2-AS1 promotes liver cancer stem cell self-renewal via BMP signaling. EMBO J. 2019;38:e101110.

92. Yuan JH, Yang F, Wang F, Ma JZ, Guo YJ, Tao QF, et al. A long noncoding RNA activated by TGF-beta promotes the invasion-metastasis cascade in hepatocellular carcinoma. Cancer Cell. 2014;25:666-81.

93. Li R, Chen M, Liu J, Shao C, Guo C, Wei X, et al. Long noncoding RNA ATB promotes the epithelial-mesenchymal transition by upregulating the miR200c/Twist1 axe and predicts poor prognosis in breast cancer. Cell Death Dis. 2018;9:1.

94. Li W, Kang Y. A new Lnc in metastasis: long noncoding RNA mediates the prometastatic functions of TGF-beta. Cancer Cell. 2014;25:557-9.

95. Wang CZ, Yan GX, Dong DS, Xin H, Liu ZY. LncRNA-ATB promotes autophagy by activating yes-associated protein and inducing autophagyrelated protein 5 expression in hepatocellular carcinoma. World J Gastroenterol. 2019;25:5310-22.

96. Hu ML, Wang XY, Chen WM. TGF-beta1 upregulates the expression of IncRNA UCA1 and its downstream HXK2 to promote the growth of hepatocellular carcinoma. Eur Rev Med Pharmacol Sci. 2018;22:4846-54.

97. Liang J, Liao J, Liu T, Wang Y, Wen J, Cai N, et al. Comprehensive analysis of TGF-beta-induced mRNAs and ncRNAs in hepatocellular carcinoma. Aging (Albany NY). 2020;12:19399-420.

98. Zhang J, Han C, Ungerleider N, Chen W, Song K, Wang Y, et al. A transforming growth factor- $\beta$ and $\mathrm{H} 19$ signaling Axis in tumor-initiating hepatocytes that regulates hepatic carcinogenesis. Hepatology. 2019;69: 1549-63.

99. Wang F, Yuan JH, Wang SB, Yang F, Yuan SX, Ye C, et al. Oncofetal long noncoding RNA PVT1 promotes proliferation and stem cell-like property of hepatocellular carcinoma cells by stabilizing NOP2. Hepatology. 2014;60:1278-90.

100. Jin J, Xu H, Li W, Xu X, Liu H, Wei F. LINC00346 acts as a competing endogenous RNA regulating development of hepatocellular carcinoma via modulating CDK1/CCNB1 Axis. Front Bioengineering and Biotechnology. 2020;8:54

101. Faivre S, Santoro A, Kelley RK, Gane E, Costentin CE, Gueorguieva I, et al. Novel transforming growth factor beta receptor I kinase inhibitor galunisertib (LY2157299) in advanced hepatocellular carcinoma. Liver Int. 2019:39:1468-77.

102. Song $S$, He X, Zeng Z, Zhang H, Yao Q, Yang F, et al. Blocking transforming growth factor-beta reduces the migration and invasion of the residual tumour after TAE. Am J Transl Res. 2019;11:2155. 
103. Zhong M, Zhong C, Cui W, Wang G, Zheng G, Li L, et al. Induction of tolerogenic dendritic cells by activated TGF- $\beta /$ Akt/Smad2 signaling in RIG-Ideficient stemness-high human liver cancer cells. BMC Cancer. 2019;19.

104. Lind H, Gameiro SR, Jochems C, Donahue RN, Strauss J, Gulley JL, et al. Dual targeting of TGF- $\beta$ and PD-L1 via a bifunctional anti-PD-L1/TGF- $\beta R$ Il agent: status of preclinical and clinical advances. J Immunother Cancer. 2020;8: e433.

105. Kelley RK, Gane E, Assenat E, Siebler J, Galle PR, Merle P, et al. A phase 2 study of Galunisertib (TGF-beta1 receptor type I inhibitor) and Sorafenib in patients with advanced hepatocellular carcinoma. Clin Transl Gastroenterol. 2019;10:e56.

106. Yao X, Zhao C, Yin H, Wang K, Gao J. Synergistic antitumor activity of sorafenib and artesunate in hepatocellular carcinoma cells. Acta Pharmacol Sin. 2020;16:1.

107. Kong J, Qiu Y, Li Y, Zhang H, Wang W. TGF- $\beta 1$ elevates P-gp and BCRP in hepatocellular carcinoma through HOTAIR/miR-145 axis. Biopharm Drug Dispos. 2019:40:70-80.

108. Takahashi K, Yan IK, Kogure T, Haga H, Patel T. Extracellular vesicle-mediated transfer of long non-coding RNA ROR modulates chemosensitivity in human hepatocellular cancer. Febs Open Bio. 2014;4:458-67.

109. Zhu J, Luo Z, Pan Y, Zheng W, Li W, Zhang Z, et al. H19/miR-148a/USP4 axis facilitates liver fibrosis by enhancing TGF-beta signaling in both hepatic stellate cells and hepatocytes. J Cell Physiol. 2019;234:9698-710.

110. Giannelli G, Villa E, Lahn M. Transforming growth factor- as a therapeutic target in hepatocellular carcinoma. Cancer Res. 2014;74:1890-4.

111. Hou ZH, Xu XW, Fu XY, Zhou LD, Liu SP, Tan DM. Long non-coding RNA MALAT1 promotes angiogenesis and immunosuppressive properties of HCC cells by sponging miR-140. Am J Physiol Cell Physiol. 2020;318:C649-63.

112. Liu L, Li N, Zhang Q, Zhou J, Lin L, He X. Inhibition of ERK1/2 signaling impairs the promoting effects of TGF- $\beta 1$ on hepatocellular carcinoma cell invasion and epithelial-Mesenchymal transition. Oncology Research Featuring Preclinical and Clinical Cancer Therapeutics. 2017;25:1607-16.

\section{Publisher's Note}

Springer Nature remains neutral with regard to jurisdictional claims in published maps and institutional affiliations.

Ready to submit your research? Choose BMC and benefit from:

- fast, convenient online submission

- thorough peer review by experienced researchers in your field

- rapid publication on acceptance

- support for research data, including large and complex data types

- gold Open Access which fosters wider collaboration and increased citations

- maximum visibility for your research: over $100 \mathrm{M}$ website views per year

At $\mathrm{BMC}$, research is always in progress.

Learn more biomedcentral.com/submissions 